\title{
Edge Subdivision Algorithm Analysis Based on the Color Linear CCD
}

\author{
Ziyang Xu, Guoxin Ma \\ School of Electronics and Information Engineering, South China University of \\ Technology, China \\ beyond-yang@foxmail.com
}

Keywords: color linear CCD; edge detection; subdivision algorithm; least-squares fitting

Abstract. Because of the linear CCD is used in non-contact dimensional measurement, especially at the edge of the detects object, the edge of the object can not be well coincided with the pixel edge of the sensor, there will be some errors. Currently, in order to reduce this errors, there are some edge subdivision algorithm based on dynamic probability subdivision method, interpolation method, fitting method and modem method and so on. Since the detection platform of this design is based on the color linear CCD, as well as taking into account the actual situation. On the basis of analysis of interpolation and fitting, an improved fitting is proposed, which is based on the most advantage points. At the same time, the design idea of this algorithm is introduced in detail, by measuring the same object, then using the above analysis of several algorithms, the experiment results show that the accuracy of the least-squares fitting algorithm based on the most advantage points is better. This result not only has important significance to improve the accuracy for CCD to detect object edge, but also provides a good basis for the application of the color linear CCD.

\section{Introduction}

Charge couple device(CCD) is a new type of photoelectric conversion image sensor. Including the application of measurement, document scanning, spectroscopy and other fields[1]. In the past, single-output linear CCD as a photoelectric sensor is used in the size measurement system, however, the CCD sensor can not perceive the direction of the measured object size thus bringing additional error for the measurement system. But triple-output color linear CCD can play a effective role in reducing such errors[2].

In addition, in modern manufacturing, the size measurement become one of the key factors to ensure product quality. Image edge is one of the most basic features of the image, it contains most of the information of one image [3], and it is an important guarantee to achieve image segmentation, image recognition and image classification. What's more the pixels subdivision technology is an important means to improve the precision of CCD measuring system[4]. Measurement technology based on CCD is not a new area and many traditional image processing technology are applied in industrial measuring system. With the gradual improvement of the precision requirement in industrial detection, the traditional edge detecting methods can't satisfy the practical needs, therefore, more and more attention has been paid on sub-pixel edge detecting and positioning. So far, there has been many sub-pixel edge subdivision methods, such as interpolation method, least square method, space quadrature and so on[5]. In order to further improve the accuracy of object edge detection, an improved algorithm of the least squares fitting based on the most advantage points is introduced in this paper. It can guarantee the high precision and high resolution at the same time.

\section{The Principle of CCD Measurement System}

\section{The Choice of CCD chip}

CCD is the media converter component part integrated by the photosensitive element. The color CCD image sensor in this design is TCD2964BFG of Toshiba in Japan which is a high sensitive and low dark current 21360 elements $\times 6$ line CCD color image sensor[6]. 
By referring to CCD driving timing data sheet, we can know that the drive timing sequence of color linear CCD we choose is more complex than others. Its output signal can be more reliable, more stable by being processed by AD9826. To design the CCD drive timing, using Verilog HDL as programming language. The soft development tool of CCD signal simulation is Quartus II. At last, we use the oscilloscope to watch and record the waveform. Figure.1 shows the waveform of the CCD.

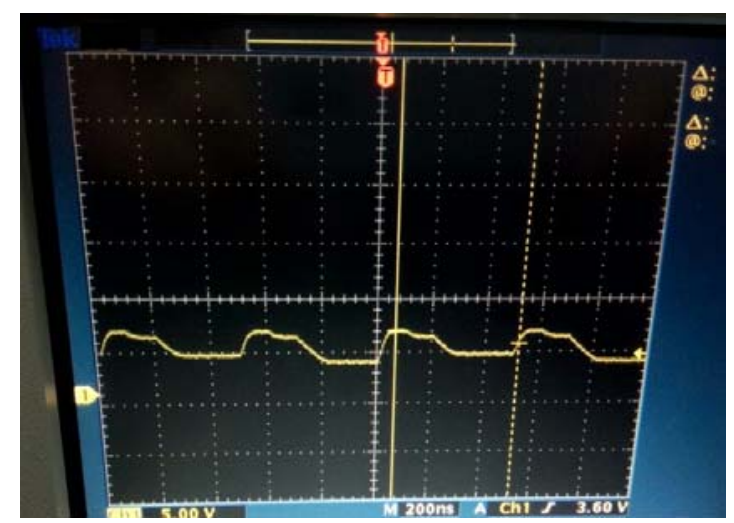

Figure.1 The output waveform of TCD2964BFG

\section{The Design of Measurement System}

Transmissive measurement techniques are widely used in detection technology of object size, which belongs to a non-contact measuring technology. The core of the system is made up of optical system, data processing system, CCD sensors and data acquisition through computer composition, but the design of the optical system often determines the measurement accuracy of the entire system to a large extent. Combined with object detection, it may be related to the edge image reconstruction, and a transmission measurement method can get better results [7], so a transmissive method for measuring is employed in this design. The corresponding principle is shown in Figure.2.

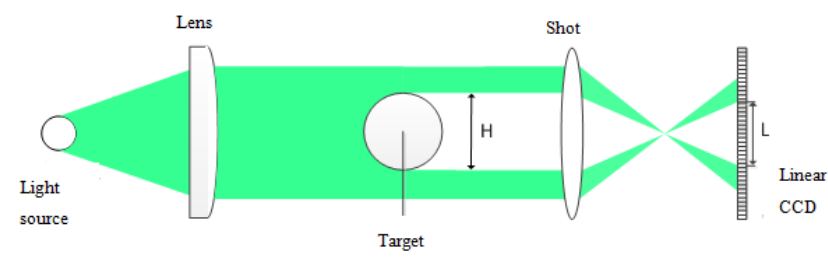

Fig.2 Schematic diagram of measurement

\section{The Principle of Edge Subdivision}

Edge segments is also called sub-pixel segmentation, sub-pixel means that there are pixels between the two physical pixels. By sub-pixel subdivision technology, we can obtain a more precise edge location of the test image[8]. When the object is projected onto the photo-voltaic device by the imaging system, the edges of the object is not a good coincidence with the cell edges of sensor, which as shown in Figure.3. Through the CCD imaging, we generally believe that the edge of the object is position 1, there is a big deviation compared with the actual position 3 , thus resulting in loss of information of object's edge. In severe cases, may lead to a margin error of \pm 0.5 pixels, but in precision instruments measurement system, the measurement error of the entire system will be even less than 0.5 pixels. In this case, the measurement results need to be optimized to improve measurement accuracy. The purpose of sub-pixel algorithm is to make measured values as close as possible to approach the edge point.

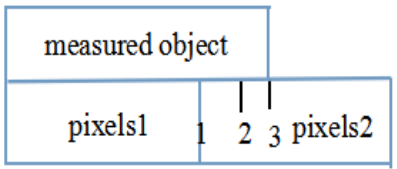

Fig.3 Schematic diagram of sub-pixel border 


\section{The Implement of Edge Subdivision Algorithm}

\section{Least Square Fitting}

Least square method is widely used in engineering fields[9], it is to fit the curve of the measuring data, then according to the existing data to obtain the function at the best position, so that make the total square error minimum, and least square fitting can easily obtain unknown data. The core idea is that if it is given $\mathrm{n}$ sets of data, such as $\left(x_{i}, y_{i}\right), i=0,1, \ldots, n-1$, we can construct m polynomial $\mathrm{p}(\mathrm{x})$. Thus the minimum of the sum $\mathrm{S}$ of square error can be calculated.

$$
S=\sum_{i=0}^{n-1}\left(y_{i}-P\left(x_{i}\right)\right)^{2},(1)
$$

Because of the edge type is unknown, the model of orthogonal polynomial is used in general, assuming orthogonal polynomials is $\left\{Q_{j}(x), j=0,1, \ldots\right\}$ based on the known $n$ points, it satisfies:

$$
\sum_{k=0}^{n-1} Q_{i}\left(x_{k}\right) Q j\left(x_{k}\right)\left\{\begin{array}{l}
=0, i \neq j \\
\neq 0, i=j
\end{array}\right.
$$

$Q_{j}(x)$ is j polynomial, $Q_{0}(x)=1$, from the orthogonal polynomial principle we can obtain $\mathrm{m}$ polynomial as follows: $Q_{m}(x)=x Q_{m-1}(x)+\sum_{i=0}^{m-1} q_{i} Q_{i}(x)$, (3)

By the formula (2), we know that $\sum_{k=0}^{n-1} x_{k} Q_{i-1}\left(x_{k}\right) Q_{j}\left(x_{k}\right)+\sum_{k=0}^{n-1} q_{j} Q_{j}\left(x_{k}\right) Q_{j}\left(x_{k}\right)=\left\{\begin{array}{l}=0, i \neq j \\ \neq 0, i=j\end{array}\right.$, (4), if $i=1$, $Q_{1}(x)=x-\alpha_{0}, \alpha_{0}=\frac{1}{n} \sum_{k=0}^{n-1} x_{k}$, (5); if $i \geq 2, Q_{m}(x)=x Q_{m-1}(x)-\alpha_{m} Q_{m-1}(x)-\beta_{m} Q_{m-2}(x)$. So the $m$ polynomial of least squares fitting can be calculated as shown in formula (6).

$$
P_{m}(x)=C_{0} Q_{0}(x)+C_{1} Q_{1}(x)+\cdots+C_{m} Q_{m}(x),(6)
$$

Through formula (1), we know that the total square error as shown in formula (7).

$$
S=\sum_{i=0}^{m} C_{i}^{2}\left(\sum_{k=0}^{n-1} Q_{i}^{2}\left(x_{k}\right)\right)-\sum_{i=0}^{m} C_{i}\left(\sum_{k=0}^{n-1} Q_{i}\left(x_{k}\right) y_{k}\right)+\sum_{k=0}^{n-1} y_{k}^{2},(7)
$$

In order to make the $\mathrm{S}$ minimum, the polynomial $\mathrm{p}(\mathrm{x})$ is calculated as shown in formula (8).

$$
P(x)=\sum_{i=0}^{m} c_{i}(x-\bar{x})^{i},(8) \quad(\bar{x} \text { is average value }) .
$$

\section{Least Square Fitting Based on The Most Advantage Points}

Because the traditional least square method sets all sample points as accurate value, the result of error is unacceptable[10]. Therefore, a least squares fitting based on the most advantage points is proposed. By using CFTool (Curve Fitting Tool) of MATLAB to analyze the fitting data at the same edge, we can get the effect of six polynomial least squares fitting based on the perfect and non-perfect edge points as shown in Figure.4. The least squares fitting of the most advantage points is better than the traditional least squares method from the figure 4 .

(a)

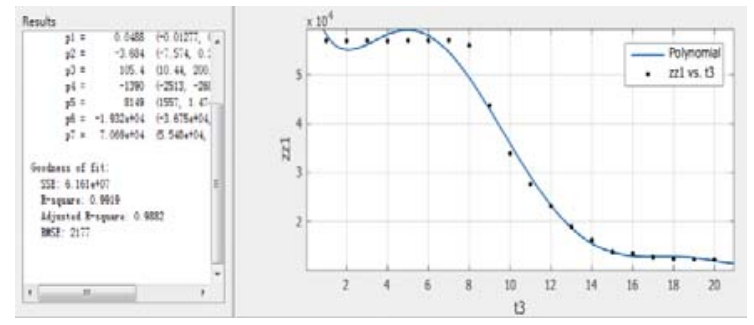

(b)

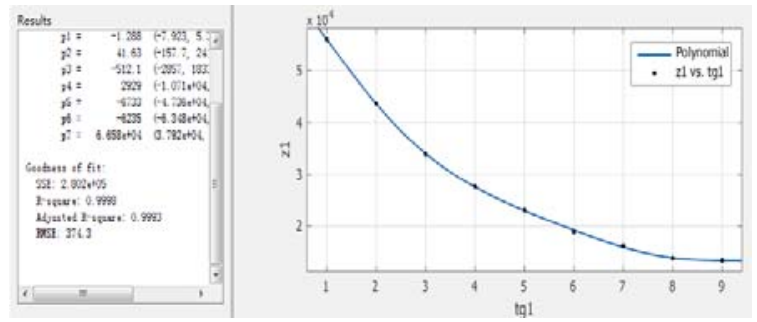

Fig.4 The comparison charts of best point and non-optimal point.(a)figure of non-optimal point fit ;(b)figure of best point fit 
Then analyzes the edge points, the two edge points of the detected object image as the center point and select 40 points according to formula (9).

$$
z(i)=\frac{|f(i)-f(i+1)|}{\min (f(i), f(i+1))},(9)
$$

The difference ratio curve of the two edge points can be calculated as shown in figure.5. As a general, supposing that the critical value is 0.05. After analyzing the data from several collection, we can get that the differential ratio at the edge will not less than 0.05 continuously.

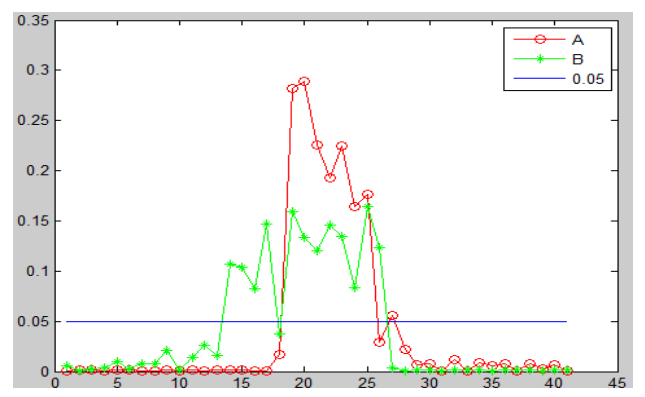

Fig.5 Edge difference ratio curve of A and B

The algorithm idea of least squares fitting based on the most advantage points is:

(1):Find out the maximum and minimum of statistical data and calculate the position of the intermediate value loc1,loc2;

(2):The interval of the most advantage points are [loc1-20,loc1+20], [loc2-20,loc2+20], according to $z(i) \geq 0.05$, after two calculates, the most advantage points of the two edges can be obtained, the flow chart is shown in figure.6;

(3):Calculate the least squares polynomial according to least squares principle;

(4): Use dichotomy to determine the position of the boundary points.

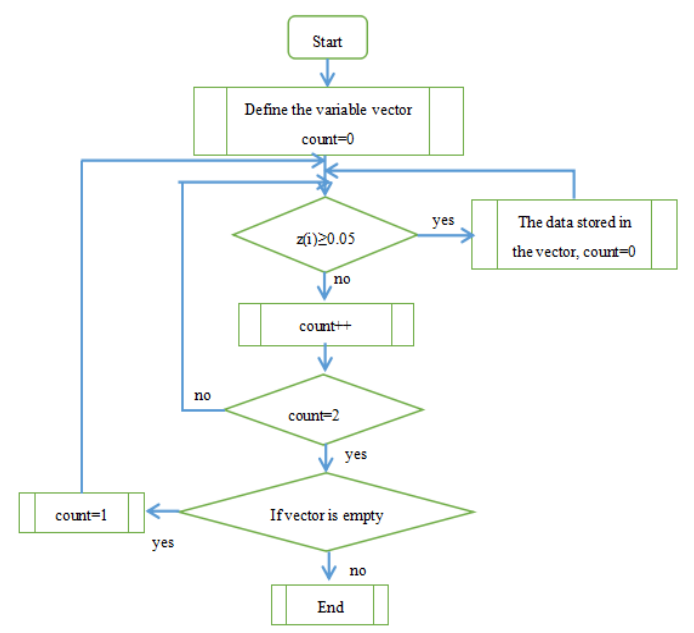

Fig.6 Flow chart of the most advantage points

\section{Experimental Analysis}

In order to verify the correctness and effectiveness of the edge subdivision algorithm that is proposed in this paper. Using C++ to program in VS2010, which measures 1000 times for the same object. The value of the object with calipers is $16.66 \mathrm{~mm}$, the results of least squares algorithm based on the non-perfect and perfect edge points are shown in figure.7(a) and (b), its average value and standard deviation as shown in table 1. 
Table.1 Compare of subdivision algorithm

\begin{tabular}{|l|c|c|}
\hline & least squares fitting & $\begin{array}{l}\text { least squares fitting based on } \\
\text { the most advantage points }\end{array}$ \\
\hline average value $(\mu \mathrm{m})$ & 16660.1224 & 16660.0819 \\
\hline standard deviation & 2.933162 & 2.221141 \\
\hline
\end{tabular}

From the figure.7(a) and (b), we can get that two kinds of results have some errors. The fluctuation range for measurement results of traditional least squares method is relatively large, however, least squares fitting based on the most advantage points can effectively reduce the volatility of the error. Moreover, the standard deviation of least squares fitting based on the most advantage points is less than traditional least squares method by table 1 .

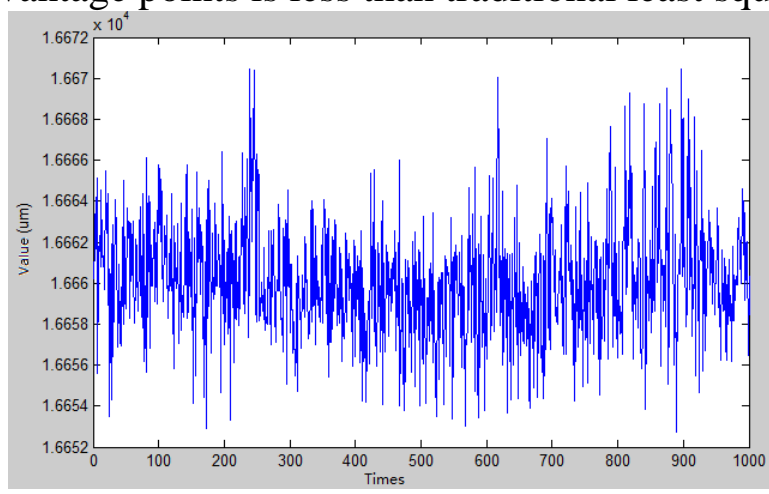

(a)

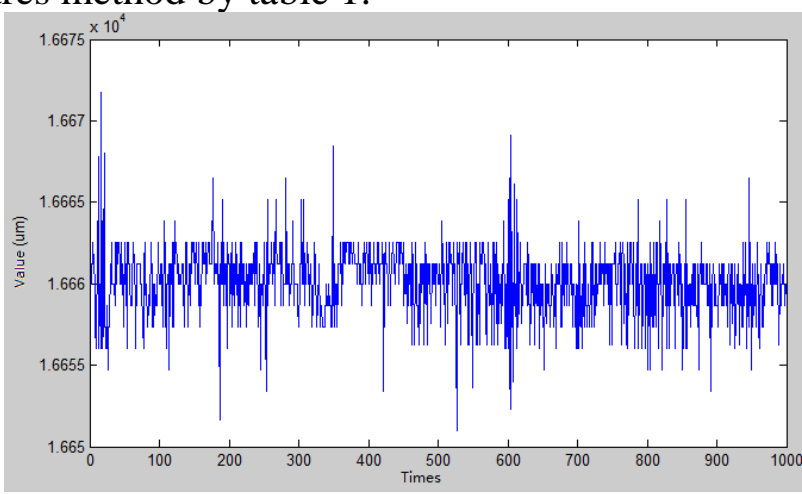

(b)

Fig.7 Measurement results chart of two kinds of edge subdivision algorithm. (a)graph of least-squares fitting; (b)graph of least-squares fitting based on the most advantage points

\section{Conclusions}

In the detecting system of non-touch measurement based on color linear CCD, the hardware circuit has been designed and a measurement system based on transmission also has been built to reduce errors in hardware design. The quality of the edge points of the CCD image affects measurement accuracy greatly, so a new subdivision algorithm technology of the least square based on the most advantage points is proposed in this paper to improve the accuracy. Experimental results indicate that it can detect the position of the edge of the image effectively and give superior performance compared with other known method. Hence, the method in this paper that introduces the application of the least squares fitting based on the most advantage points in the edge detecting will have a bright future in several industrial measurements.

\section{References}

[1] James R. Janesick, “Scientific Charge-Coupled Devices,” SPIE Publications, 2001.

[2] Zhang Shengbin,Wang Qingyou,Guo Qing.Journal of Optoelectronics • Laser,2001,12 (11):1159-1161. (In Chinese)

[3] Peng Fengfu,Huang Long,Wang Chengjie etal. Computer and Digital Engineering,2010, 38(12):118-119. (In Chinese)

[4] Su Xiaogang, Hu Xiaodong, Zhang Xiaodong. “A Single-Ring Absolute Encoder Subdivision Method,” Measuring Technology and Mechatronics Automation, International Conference. 2015,pp.55-58. 
[5] Lv Hailei,Lei Zhiyong,Wang Zemin.Science Technology and Engineering,2008,8(3)634-635. (In Chinese)

[6] TOSHIBA.TCD2964BFG datasheet[Z].2008.

[7] Zhang Limin,Gao Feng, Li Jiao,et al.CHINESE JOURNAL OF LASER, 2009,36(10):25532556. (In Chinese)

[8] Xu Guosheng. “Sub-pixel Edge Detection Based on Curve Fitting,” Information and Computing Science, International Conference. 2009,pp.373-375.

[9] Tian Long,Liu Zongtian.Computer Science,2012,39(6A):482-484. (In Chinese)

[10] Ma Xiangnan,Li Hang,Liu Lili,et al.Journal of Henan University of Science and Technology: Natural Science ,2014,35(3):19-21. (In Chinese) 